\title{
Benefits of participating in mastermind groups
}

\author{
Pernilla Garmy ${ }^{1,2 *}$, Ulrika Olsson Möller ${ }^{2}$, Cecilia Winberg'1,3, Lina Magnusson ${ }^{1,4}$ and Nelli Kalnak $^{5}$ \\ ${ }^{1}$ Department of Health Sciences, Medical Faculty, Lund University, Sweden \\ ${ }^{2}$ Faculty of Health Sciences, Kristianstad University, Sweden \\ ${ }^{3}$ Ystad hospital, Region Skåne, Sweden \\ ${ }^{4}$ Department of Public Health and Community Medicine, Section of Social Medicine, Sahlgrenska Academy, Gothenburg University, Sweden \\ ${ }^{5}$ Child and Adolescent Psychiatry Unit, Department of Clinical Sciences, Medical Faculty, Lund University, Sweden
}

\begin{abstract}
The academic career path is seldom straightforward. Many health professionals and researchers face stress and uncertain employment opportunities. Joining a collegial support group, a so-called "mastermind" group, is one way to help navigate these challenges. We investigated postdoctoral researchers' (N=16) experiences with participating in a mastermind group using an online survey. Four themes emerged from their responses: (I) A place that offers conversation in confidence; (II) An opportunity for personal and professional development; (III) A quality break and time for reflection, and (IV) Challenges experienced by mastermind group participants. This study establishes that taking part in a mastermind group can effectively help shift focus from the negative aspects of a challenge faced by its group members to the positive aspects of a potential solution.
\end{abstract}

\section{Introduction}

The academic career path is seldom straightforward. Many health professionals and researchers face stress and uncertain employment opportunities. This experience motivates many to abandon health care and/ or academia [1]. Participating in a collegial or peer-mentoring network (e.g., a mastermind group) represents one way of managing the challenges associated with working in health care and academia.

Expectations and challenges can be demanding for early career health professionals and researchers. Faculty support is not always available and often fails to meet specific individual needs [2]. Mentoring is traditionally based on one-to-one hierarchical relationships, e.g., new faculty members being mentored by senior ones. However, more recent models of mentorship in academia suggest forming peer-mentoring networks composed of non-hierarchical partners [3]. The value of peer-mentoring networks for early career academics lies in extending beyond their own institution/department's walls, as emphasized in Pegg et al. [4]. A network based on reciprocal partnerships with university colleagues who are not involved in the same institution/department can provide a positive and non-dependent context for mentorship. Pegg and colleagues highlight the importance of formalizing informal peermentoring networks and they recommend meeting regularly, setting goals, defining how to meet (in person or online), and consciously determining the size and composition of the group.

A "mastermind" group is a peer-mentoring network usually composed of three to six participants with a maximum of eight to ten participants. The main purpose of a mastermind group is to help its members navigate the challenges and solve problems using their collective knowledge and experiences. These groups are self-directed. The original purpose of the mastermind group concept was to enable a group-based approach to problem-solving [5]. Each mastermind group agrees on the agenda, goals, approaches, timelines, frequency, and format of its meetings. Meetings are regular and can be face-to-face, virtual (e.g., via teleconference or Skype), or a mixture of both based on the group members' needs and preferences. The agenda of the meetings address a specific common interest of the participants, e.g., research or teaching [6]. The members support each other by contributing different skills, perspectives, and experiences. Mentoring programs with colleagues at a medical faculty can lead to participants experiencing an appreciative culture, clarifying their own career goals and priorities, and feeling enhanced enthusiasm for collaboration [7]. The success of peer-mentoring programs in a university setting has been reported to rely on the relationships within the group and on the use of feedback as a coaching tool [7].

\section{Methods}

\section{Design}

This study was conducted using an empirical qualitative approach. Qualitative research methodologies aim to describe and understand subjective experiences [8]. The selection of this research design was dependent on the nature of the issue being addressed. A survey with open-ended questions was used to provide answers to questions concerning early career researchers experiences with participating in a mastermind group. Next, we performed a qualitative content analysis because this method describes variations among experiences and distinguishes between their differences and similarities [9].

\section{Sample and data collection}

In December 2018, an online survey with information about the study and its voluntary nature was sent to 100 early career researchers

${ }^{\star}$ Correspondence to: Pernilla Garmy, Department of Health Sciences, Medical Faculty, Lund University, Sweden, E-mail: pernilla.garmy@med.lu.se

Key words: collegial support, mastermind group, peer-mentoring network, higher education, postdoctoral fellow

Received: March 26, 2019; Accepted: April 03, 2019; Published: April 05, 2019 
who had participated in the university post-doctoral career program at a Swedish university, from which the opportunity to join a mastermind group was offered. The participation was anonymous.

The following background information was collected: age, sex, faculty of the participant, the duration of their participation in the mastermind group, how often the meetings took place, if the mastermind meetings were in person and/or online, and the number of participants in the group. The survey also included the following openended questions:

- Please describe your experiences of participating in a mastermind group.

- Please give examples of topics discussed in the mastermind group meetings.

- Please describe challenges as well as positive experiences of participating in a mastermind group.

\section{Analysis}

Conventional qualitative content analysis was used, specifically an inductive approach [10]. The text material was analyzed using qualitative content analysis focused on both the manifest content and the latent content because the purpose of the study was to describe and understand the early career researchers' experiences. This approach is appropriate when describing differences and similarities in a textual format [9]. This analysis was executed by all of the authors by reading the responses to the open-ended questions several times to achieve comprehension. The meaning units that responded to the purpose were condensed by the first and last authors, which involved summarizing the content without losing its core meaning. This codification method enables understanding context through its relationship to the text. All of the authors met to compare their codifications and discuss the similarities and differences. Similar codes were highlighted and formed four themes $[9,10]$.

\section{Ethical considerations}

Before participants were recruited, a research ethics application was approved by Kristianstad University, Sweden (2018-232-624). All procedures were conducted in accordance with the Declaration of Helsinki.

\section{Results}

The survey was completed by 16 early career researchers aged 29-47 years; 13 women and three men. The faculty of medicine was represented by nine participants and the faculty of natural sciences by seven. The duration of their participation in a mastermind group

Table 1. List of topics discussed in the mastermind meetings

\begin{tabular}{|l|}
\hline Topics \\
\hline The scientific writing process and publication strategies \\
\hline Teaching strategies \\
\hline Authorship on publications \\
\hline Work-life balance \\
\hline Grant and fellowship applications \\
\hline Job applications, interviews, and tests \\
\hline Leadership skills \\
\hline Short- and long-term career goals \\
\hline Navigating and networking in the research community \\
\hline Productivity and time management \\
\hline
\end{tabular}

varied from four months to two years and the frequency of the meetings ranged from once per month to every third month. Only one participant reported that the mastermind meetings were mainly conducted online (Skype) and all of the others reported that the meetings were in person. The number of members per group ranged from three to six.

\section{Topics discussed in the mastermind meetings}

The topics discussed by the different groups all focused on how to navigate in academia as an early career researcher (Table 1).

Four themes describing experiences were identified through content analysis of the answers to the open-ended questions: (I) A place that offers conversations in confidence; (II) Personal and professional development; (III) A quality break and time for reflection, and (IV) Challenges experienced by mastermind group participants. The themes are exemplified with quotations, presented below.

\section{Theme (I): A place that offers conversation in confidence}

The participants described the importance of having a safe place to engage in conversations in confidence. They valued having a context in which both personal success and failures were discussed in a warm, non-judgmental, and friendly way.

"To me, this network is a safe and friendly place that is stimulating and creative. An opportunity to reflect my reality in others."

"It has been valuable with a small forum with the opportunity to speak freely, i.e., in confidence, about what is relevant, both disappointments and success."

"The meetings have been a 'safe spot' where I have been given the opportunity to express thoughts that I otherwise did not have a clear forum for."

\section{Theme (II): Opportunity for personal and professional development}

The second theme highlights that the mastermind group meetings contributed to both the personal and professional development of its members. The participants reported that they felt inspired and motivated to visualize their future career more explicitly and that they gained new insight about themselves during the mastermind meetings:

"I have been able to think a bit higher, dare to dream a bit bigger, and have space to share both difficult and fun experiences safely."

"It gave me the opportunity to dedicate time to thinking about my career, something I would have considered a luxury otherwise."

\section{Theme (III): "A quality break and time for reflection"}

The participants expressed that the mastermind meetings provided quality time for reflection as well as opportunities to learn from others' experiences.

\section{"I really like this breathing hole that provides me with new energy."}

"Creating a sense of community and lightning the feelings of insecurity by sharing them and hearing them echoed in others."

"...getting opinions from outside my narrow field is excellent considering I want a career in science management. Their advice has been invaluable." 


\section{Theme (IV) Challenges experienced by mastermind group participants}

The most common challenge reported by the participants was scheduling the meetings. It was often difficult to identify a time when all of the members would be able to attend. Other challenges concerned trust issues, i.e., the fear of a lack of confidentiality and the worry of personal information being spread outside of the group. Other negative experiences related to the composition of the group, which included: feeling excluded by subgroup formation based on, e.g., having the same profession or being at the same career level; unbalanced gender composition, e.g., being the only man in the group; and, lastly, a dominant group member obstructing the aim of the meetings.

"It was sometimes difficult to agree on an appointment because of our busy schedules."

"Originally, we thought that we would plan in advance the topics to discuss at the upcoming meetings but this didn't happen. Oftentimes, we would decide on the same day. It did work out and it worked for us, but going forward, it might be worth trying to plan ahead."

"The other three participants come from somewhat more similar and very technical fields than me, and we did not have an equal gender balance with three male and one female group member.

\section{Discussion and conclusion}

A mastermind group is a peer-mentoring network that contributes to a deeper understanding of each member's current work situation, experiences, and skills. The members collectively tackle current challenges and opportunities, mentor one another, and hold each other accountable for actions collectively agreed upon at group meetings. These groups can be effective toward shifting focus from the negative aspects of a challenge faced by a group member to the positive aspects of a potential solution. Involvement in a mastermind group facilitates early career researchers' pursuit of a career within or outside academia. The mastermind group provides a level of support beyond that offered by the department/institution. In the long-term (median four years), involvement in peer mentoring activities has been associated with increased satisfaction with academic skills (e.g. scientific writing) and a sustainable effect on academic productivity [11].

Pegg et al. [4] suggest that peer-mentoring networks can help to identify key aspects required to move the academic career forward and the people and resources best-suited to support an individual's professional needs; the data in the present paper support both of these. Furthermore, the importance of confidentiality was highlighted by both the positive and negative experiences of the participants in the present study.

The current study is limited to a small sample of participants from only one university. However, previous studies describing Mastermind groups in a medical academic context are lacking. Future studies in this area may elucidate which kind of coaching tools peer mentoring groups use, and explore their effectiveness in moving the career forward.

\section{References}

1. Garforth L, Kerr A (2009) Constructing Careers, Creating Communities: findings of the UK KNOWING research on knowledge, institutions and gender. University of Leeds.

2. Bottoms S, Pegg J, Adams A, Wu K, Smith Risser H, et al. (2013) Mentoring from the outside: The role of a peer mentoring community in the development of early career education faculty. Mentoring \& Tutoring: Partnership in Learning 21: 195-218.

3. Sorcinelli MD, Yun J (2007) From mentor to mentoring networks: Mentoring in the new academy. Change: The Magazine of Higher Learning 39: 58-61.

4. Pegg JM, Adams AE, Risser HS, Bottoms SI, Kern AL, et al. (2014) Finding FRiENDs: Creating a community of support for early career academics. Brock Education Journal p: 24.

5. Hill N (2011) Think and grow rich: Hachette UK.

6. Fritsches K (2018) Postdoc Career Success.

7. Pololi LH, Evans AT (2015) Group Peer Mentoring: An Answer to the Faculty Mentoring Problem? A Successful Program at a Large Academic Department of Medicine. J Contin Educ Health Prof 35: 192-200. [Crossref]

8. Polit DF, Beck CT (2016) Nursing research: generating and assessing evidence for nursing practice. Philadelphia: Wolters Kluwer.

9. Schreier M (2012) Qualitative content analysis in practice: Sage Publications.

10. Hsieh HF, Shannon SE (2005) Three approaches to qualitative content analysis. Qual Health Res 15: 1277-1288. [Crossref]

11. Mayer AP, Blair JE, Ko MG, Patel SI, Files JA (2014) Long-term follow-up of a facilitated peer mentoring program. Med Teach 36: 260-266. [Crossref]

Copyright: (C2019 Garmy P. This is an open-access article distributed under the terms of the Creative Commons Attribution License, which permits unrestricted use, distribution, and reproduction in any medium, provided the original author and source are credited. 